\title{
低共熔溶剂中新型螺环吲哚衍生物的绿色合成
}

\author{
严 楠*,a,b 熊云奎 ${ }^{b}$ 夏剑辉 $b$ 苪培欣 ${ }^{b}$ \\ 雷志伟 $a$ 廖维林*, $a$ 熊 斌 $a, b$ \\ ( ${ }^{a}$ 国家单糖化学合成工程技术研究中心 南昌 330027) \\ $\left({ }^{b}\right.$ 江西师范大学化工研发中心 南昌 330027)
}

\begin{abstract}
摘要 在低共熔溶剂氯化胆碱/草酸催化作用下, 由醛、吲哚和 6,10-二氧杂螺[4.5]十烷-7,9-二酮三组分多米诺 Knoevenagel-Michael 反应合成了一系列新型螺环吲哚衍生物，产率为 $70 \% \sim 97 \%$. 该方法条件温和，操作简单，反应时 间短, 对环境友好等优点, 且催化剂廉价易得.

关键词 低共熔溶剂; 多米诺反应; 螺环吲哚衍生物; 绿色合成
\end{abstract}

\section{Green Synthesis of Novel Spirooxindole Derivatives in Deep Eutectic Solvent}

\author{
Yan, $\operatorname{Nan}^{*, a, b}$ \\ Xiong, Yunkui ${ }^{b}$ \\ Xia, Jianhui ${ }^{b}$ \\ Rui, Peixin ${ }^{b}$ \\ Lei, Zhiwei $^{a}$ \\ Liao, Weilin*,a \\ Xiong, Bin ${ }^{a, b}$ \\ ( ${ }^{a}$ National Monosaccharide Chemical Synthesis Engineering Research Center, Nanchang 330027) \\ ( ${ }^{b}$ Chemical Research and Development Center, Jiangxi Normal University, Nanchang 330027)
}

\begin{abstract}
A series of novel spirooxindole derivatives were synthesized by three-component domino Knoevenagel-Michael reaction between aldehydes, indole and 6,10-dioxaspiro[4.5]decane-7,9-dione using the deep eutectic solvent (DES) choline chloride-oxalic acid as a catalyst in 70\% 97\% yields. This method has some distinct advantages such as easy work-up, mild reaction conditions, short reaction time, environmental friendliness and accessibility of catalyst.
\end{abstract}

Keywords deep eutectic solvent; domino reaction; spirooxindole derivatives; green synthesis

多米诺反应(domino reaction)是指在特定反应条件 下，发生两个或者两个以上键(通常为碳一碳键)形成的 反应. 该反应过程中, 不需额外添加试剂或催化剂, 中 间体也无需分离和提纯，与传统的多步合成方法相比， 多米诺反应能够大大地提高反应效率和原子利用

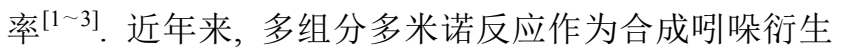
物的有效合成方法已经得到有机合成化学家的广泛关 注 $^{[4 \sim 6]}$.

螺环吲哚衍生物是一类重要的杂环化合物, 具有显 著的生物活性和应用功能 ${ }^{[7]}$. 它也是合成 $\beta$-取代的色氨 酸、稠杂环色胺、具有生物活性的咔啉和咔唑衍生物及 抗肿瘤药物 ${ }^{[8 \sim 10]}$ 的重要中间体, 该类化合物的合成已成 为当前的研究热点. 这类化合物的合成最早由
Yonemitsu 小组 ${ }^{[11]}$ 报道，即用酫、2,2-二甲基-1,3-二啞 烷-4,6-二酮(米氏酸)、吲哚三组分在乙腈溶液中一锅法 反应制备, 但存在时间较长, 收率不高, 易生成双取代 的缩合产物-Michael 加成产物等缺点. 近年来, 已出现 采用脯氨 酸 ${ }^{[12,13]}, \mathrm{TiCl}_{4} / \mathrm{Et}_{3} \mathrm{~N}^{[14]}$,三氟甲磺酸 镱 ${ }^{[7]}$, $\mathrm{Cu}(\mathrm{OAc})_{2} / \mathrm{KH}_{2} \mathrm{PO}_{4}{ }^{[15,16]}$, 超声辅助 ${ }^{[17]}$, 固相合成 ${ }^{[18]}$ 等来 改进 Yonemitsu 反应缩合反应的报道. 这些合成方法具 有一定的潜在应用价值，但存在反应时间长、后处理繁 琐、产率低、底物普适性不好等缺点. 因此, 发展绿色、 简便和高效合成的新方法势在必行.

近年来, 胆碱类低共熔溶剂(deep eutectic solvent, DES)由于具有低挥发性、低熔点、高热稳定性、低毒可 降解和可设计性等优点作为一类新型的绿色溶剂引起

* E-mail: yannan104@163.com

Received September 13, 2014; revised November 13, 2014; published online November 21, 2014.

Project supported by the National Science and Technology Support Program of China (No. 2009BAI75B02), the Innovation Fund Designated for Graduate Students of Jiangxi Province (No. YC2014-B030), and the Outstanding Doctoral Dissertation Cultivation Program of Jiangxi Normal University.

国家科技支撑计划(No. 2009BAI75B02)、江西省研究生创新基金(No. YC2014-B030)、江西师范大学优秀博士论文培育计划资助项目. 
了人们的广泛关注 ${ }^{[18 ~ 21]}$. 目前, 胆碱类低共熔溶剂作为 溶剂和催化剂广泛应用于各种有机反应, 如双吲哚类烷 基化合物 ${ }^{[2]}$ 及其双(4-羟基香豆素)甲烷类化合物合成研 究 ${ }^{[23]}$, Knoevenagel 缩合反应合成橙酮类化合物 ${ }^{[24]}$,三 组分合成螺环静红类化合物 ${ }^{[25]}$. 尽管 DES 已经被广泛 用于多种有机反应, 但是在螺环吲哚衍生物的合成中还 没有报道过. 基于此, 本文报道采用氯化胆碱/草酸 $(\mathrm{ChCl} / \mathrm{Ox})$ 低共熔溶剂为反应介质和催化剂, 利用自制

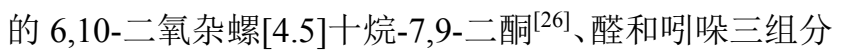
“一锅法” 合成了 13 种新型螺环吲哚衍生物, 合成路线 如 Scheme 1.

\section{1 结果与讨论}

\section{1 反应条件优化}

为了优化反应条件, 以苯甲醛、吲哚和 6,10-二氧杂 螺[4.5]烷-7,9-二酮为反应模型, 考察了不同催化剂、 溶剂、催化剂用量对 $\mathbf{4 a}$ 产率的影响. 如表 1 所示, 在传 统催化剂如 $\mathrm{TsOH}, L$-Proline, $\mathrm{Yb}(\mathrm{OTf})_{3}$ 中, 虽然也能得 到预期的产物, 但反应时间长, 收率较低(表 1, Entries $1 \sim 3)$. 接着考察了四种胆碱类低共熔溶剂 - -氯化胆 碱/尿素 $(\mathrm{ChCl} / \mathrm{urea})$ 、氯化胆碱/氯化镧 $\left(\mathrm{ChCl} / \mathrm{LaCl}_{3}\right.$ ・ $\left.6 \mathrm{H}_{2} \mathrm{O}\right)$ 、氯化胆碱/对甲苯磺酰胺 $(\mathrm{ChCl} / \mathrm{PTSA}) 、$ 氯化胆 碱/草酸 $(\mathrm{ChCl} / \mathrm{Ox}$ )催化效果(表 1, Entries 5 10), 其中 $\mathrm{ChCl} / \mathrm{Ox}$ 催化效果最佳, 在室温下, 反应 $40 \mathrm{~min}$ 后, 产 率达到 91\% (表 1, Entry 7). 室温无溶剂条件下，反应时 间缩短, 且产率进一步提高, 达到 95\%(表 1, Entry 8). 为了优化 $\mathrm{ChCl} / \mathrm{Ox}$ 的用量, 结果发现, 当减少 $\mathrm{ChCl} / \mathrm{Ox}$ (表 1, Entry 9)使用量, 产率降低; 当补加 $\mathrm{ChCl} / \mathrm{Ox}$ (表 1, Entry 10)使用量, 反应时间缩短, 但是产率有降低. 我 们还尝试增加反应剂量到 $30 \mathrm{mmol}$, 在相同条件下 $\mathrm{ChCl} / \mathrm{Ox}$ 依然能顺利完成上述模型反应. 说明 $\mathrm{ChCl} / \mathrm{Ox}$ 体系有望用于实际合成中(表 1, Entry 11).

\section{表 1 螺环吲哚衍生物合成条件的优化 ${ }^{a}$}

Table 1 Optimization of reaction conditions for synthesizing spirooxindole derivatives

\begin{tabular}{|c|c|c|c|c|}
\hline Entry & Catalyst & Solvent & Time & Yield $^{b} / \%$ \\
\hline 1 & $\mathrm{TsOH}(5 \mathrm{~mol} \%)$ & $\mathrm{CH}_{3} \mathrm{CN}$ & $8 \mathrm{~h}$ & 48 \\
\hline 2 & $L$-Proline (5 mol\%) & $\mathrm{CH}_{3} \mathrm{CN}$ & $8 \mathrm{~h}$ & 55 \\
\hline 3 & $\mathrm{Yb}(\mathrm{OTf})_{3}(5 \mathrm{~mol} \%)$ & $\mathrm{CH}_{3} \mathrm{CN}$ & $8 \mathrm{~h}$ & 45 \\
\hline 4 & $\mathrm{ChCl} /$ urea $(1 \mathrm{~mL})$ & Ethanol & $6 \mathrm{~h}$ & Trace \\
\hline 5 & $\begin{array}{l}\mathrm{ChCl} / \mathrm{LaCl}_{3} \cdot 6 \mathrm{H}_{2} \mathrm{O} \\
(1 \mathrm{~mL})\end{array}$ & Ethanol & $6 \mathrm{~h}$ & 10 \\
\hline 6 & $\mathrm{ChCl} / \mathrm{PTSA}(1 \mathrm{~mL})$ & Ethanol & $6 \mathrm{~h}$ & Trace \\
\hline 7 & $\mathrm{ChCl} / \mathrm{Ox}(1 \mathrm{~mL})$ & Ethanol & $40 \mathrm{~min}$ & 91 \\
\hline 8 & $\mathrm{ChCl} / \mathrm{Ox}(1 \mathrm{~mL})$ & Solvent free & $20 \mathrm{~min}$ & 95 \\
\hline 9 & $\mathrm{ChCl} / \mathrm{Ox}(0.8 \mathrm{~mL})$ & Solvent free & $30 \mathrm{~min}$ & 92 \\
\hline 10 & $\mathrm{ChCl} / \mathrm{Ox}(1.5 \mathrm{~mL})$ & Solvent free & $15 \mathrm{~min}$ & 94 \\
\hline 11 & $\mathrm{ChCl} / \mathrm{Ox}(30 \mathrm{~mL})^{c}$ & Solvent free & $20 \mathrm{~min}$ & 95 \\
\hline
\end{tabular}

\section{$1.2 \mathrm{ChCl} / \mathrm{Ox}$ 低共熔物对不同底物的普适性}

在优化反应条件基础上, 我们以吲哚、6,10-二氧杂 螺 [4.5]十烷-7,9-二酮为底物, 研究了醛的适用范围, 如 表 2 所示, 无论是芳香醛, 还是脂肪酫均能够顺利地参 与反应(除邻硝基苯甲醛), 均可以中等以上产率得到目 标产物. 从表 2 结果可以看出, 吸电子芳香醛产物产率 普遍高于给电子基产物收率; 另外实验发现芳香醛反应 速度快于脂肪酫，带吸电子基的芳香醛反应速度快于带 供电子基的芳香醛，对硝基苯甲醛反应较快, $20 \mathrm{~min}$ 后, 产率高达 97\%\% (表 2, Entry 2); 而对甲氧基苯甲醛活性 较差，反应 $40 \mathrm{~min}$ 后，产率为 $74 \%$ (表 2, Entry 7). 这可 能是由于苯环上的吸电子基团使羰基上带有更多的正 电荷, 有利于亲核加成反应的进行. 此外, 苯甲醛上取 代基的位置似乎对产物收率有一些影响, 用邻硝基苯甲 醛作底物时表现更明显，由于空间位阻太大，仅得到 Knoevenagel 缩合反应产物(40), 而空间位阻较小的芳 香酫，分别以 $81 \% \sim 91 \%$ 收率得到目标产物(表 2, Entries 8〜11).

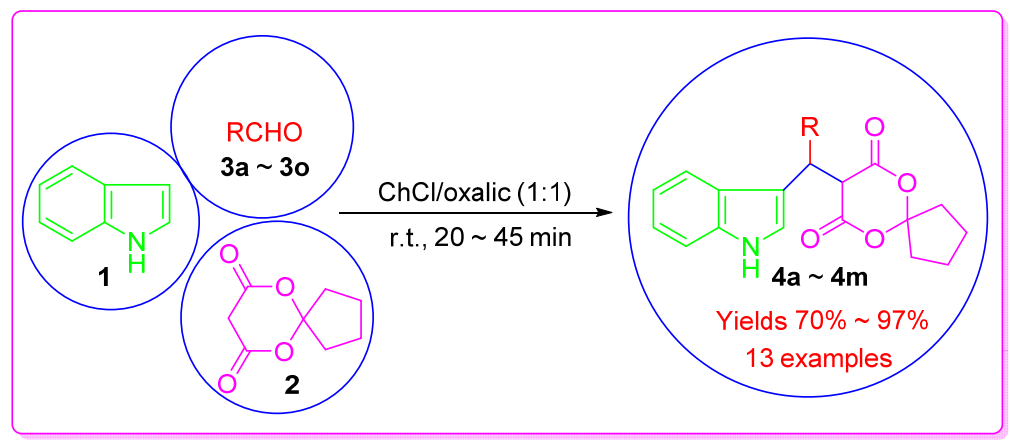

图式 1 低共熔溶剂中新型螺环吲哚衍生物合成路线

Scheme 1 Synthetic routes of novel spirooxindole derivatives in deep eutectic solvent 
表 $2 \mathrm{ChCl} / \mathrm{Ox}$ 催化合成螺环吲哚衍生物

Table 2 Synthesis of spirooxindole derivatives catalyzed by $\mathrm{ChCl} / \mathrm{Ox}$

\begin{tabular}{|c|c|c|c|c|c|}
\hline Entry & $\mathrm{R}$ & Yield $^{a} / \%$ & $\mathrm{me} / \mathrm{n}$ & roduct & t m.p..$^{b}{ }^{\circ} \mathrm{C}$ \\
\hline 1 & $\mathrm{C}_{6} \mathrm{H}_{5}$ & 95 & 20 & $4 a$ & $139 \sim 140$ \\
\hline 2 & $4-\mathrm{NO}_{2} \mathrm{C}_{6} \mathrm{H}_{4}$ & 97 & 20 & $4 b$ & $122 \sim 124$ \\
\hline 3 & $4-\mathrm{FC}_{6} \mathrm{H}_{4}$ & 88 & 25 & $4 c$ & $127 \sim 129$ \\
\hline 4 & $4-\mathrm{ClC}_{6} \mathrm{H}_{4}$ & 92 & 25 & $4 d$ & $117 \sim 119$ \\
\hline 5 & 4- $\mathrm{BrC}_{6} \mathrm{H}_{4}$ & 91 & 25 & $4 e$ & $124 \sim 126$ \\
\hline 6 & 4- $\mathrm{CH}_{3} \mathrm{C}_{6} \mathrm{H}_{4}$ & 85 & 25 & $4 f$ & $131 \sim 133$ \\
\hline 7 & 4- $\mathrm{CH}_{3} \mathrm{OC}_{6} \mathrm{H}_{4}$ & 74 & 40 & $4 g$ & $125 \sim 127$ \\
\hline 8 & $2-\mathrm{FC}_{6} \mathrm{H}_{4}$ & 91 & 25 & $4 h$ & $133 \sim 135$ \\
\hline 9 & $2-\mathrm{CH}_{3} \mathrm{OC}_{6} \mathrm{H}_{4}$ & 83 & 25 & $4 \mathbf{i}$ & $128 \sim 130$ \\
\hline 10 & $2,5-\left(\mathrm{CH}_{3} \mathrm{O}\right)_{2} \mathrm{C}_{6} \mathrm{H}_{3}$ & 81 & 35 & $4 j$ & $145 \sim 147$ \\
\hline 11 & $3-\mathrm{NO}_{2} \mathrm{C}_{6} \mathrm{H}_{4}$ & 86 & 30 & $4 k$ & $124 \sim 126$ \\
\hline 12 & $\mathrm{CH}_{3}\left(\mathrm{CH}_{2}\right)_{2}$ & 72 & 45 & 41 & $127 \sim 129$ \\
\hline 13 & $\mathrm{CH}_{3}\left(\mathrm{CH}_{2}\right)_{3}$ & 70 & 45 & $4 \mathrm{~m}$ & $106 \sim 108$ \\
\hline
\end{tabular}

${ }^{a}$ Isolated yield; ${ }^{b}$ melting points are uncorrected.

\section{$1.3 \mathrm{ChCl} / \mathrm{Ox}$ 重复使用性能}

相比于其他反应体系, 该体系的优势在于该体系的 “绿色化”。该低共熔物不仅具有原料易得、合成简单, 同时该体系还能实现回收和重复利用. 在最佳反应条件 下，以苯甲醛、吲哚和 6,10-二氧杂螺[4.5]十烷-7,9-二酮 为探针反应, 考察了 $\mathrm{ChCl} / \mathrm{Ox}$ 的重复使用性能, $\mathrm{ChCl} / \mathrm{Ox}$ 重复使用结果见表 3. 由表 3 可知, 回收后的催化剂可 以循环使用 5 次, 且其催化活性没有显著降低.

表 3 重复利用的 $\mathrm{ChCl} / \mathrm{Ox}$ 对 $\mathbf{4 a}$ 产率的影响

Table 3 Effects of $\mathrm{ChCl} / \mathrm{Ox}$ recycling on the yield of $4 \mathbf{a}$

\begin{tabular}{ccc}
\hline Run & Time/min & Yield $/ \%$ \\
\hline Fresh & 20 & 95 \\
1 & 20 & 95 \\
2 & 20 & 95 \\
3 & 23 & 94 \\
4 & 25 & 93 \\
5 & 28 & 91 \\
\hline
\end{tabular}

\section{2 结论}

在室温条件下，于 $\mathrm{ChCl} / \mathrm{Ox}$ 低共熔溶剂中，简洁、 绿色、高效地合成了一系列新型螺环衍生物, $\mathrm{ChCl} / \mathrm{Ox}$ 是一种非常有效的反应介质. 该方法优点在于 $\mathrm{ChCl} / \mathrm{Ox}$ 低共熔物原料易得、且对环境友好并可循环使用, 符合 绿色化学的发展原则, 同时为合成型螺环衍生物提供了 一种简便的合成.

\section{3 实验部分}

\section{1 仪器与试剂}

6,10-二氧杂螺[4.5]十烷-7,9-二酮按文献[24]制备, 苯甲醛、对硝基苯甲醛、2-硝基苯甲醛、间硝基苯甲醛、
对氯苯甲醛、邻氟苯甲醛、邻甲氧基苯甲醛、对甲基苯 甲醛、对甲氧基苯甲醛、2,5-二甲氧基苯甲醛、对氟苯 甲醛、对溴苯甲醛、正丁醛、正戊醛为分析纯; 其它均 为化学纯.

瑞士 BuchiB-540 型显微熔点仪(温度计未经校正); 瑞士 Bruker400 $\mathrm{MHz}$ 型核磁共振仪 $\left(\mathrm{CDCl}_{3}\right.$ 或 DMSO- $d_{6}$ 为溶剂, TMS 为内标); 日本岛津 FT-IR-8400 型红外仪 (KBr 压片); Finnigan MAT-8430 型高分辨质谱仪.

\section{2 氯化胆碱/草酸 $(\mathrm{ChCl} / \mathrm{Ox})$ 低共熔物的制备}

在 $250 \mathrm{~mL}$ 圆底烧瓶中加入氯化胆碱 $(70 \mathrm{~g}, 0.5 \mathrm{~mol})$, 草酸(45 g, $0.5 \mathrm{~mol}$ ), 升温至 $80{ }^{\circ} \mathrm{C}$ (油浴), 磁力搅拌 30 $\min$, 缓慢冷却至室温, 形成无色透明室温低共熔物 ${ }^{[22]}$. 真空干燥后待用，产率 100\%.

\section{3 螺环吲哚衍生物 $4 \mathrm{a} \sim 4 \mathrm{~m}$ 的合成}

室温条件下, 在 $10 \mathrm{~mL}$ 长试管中依次加入 6,10-二 氧杂螺[4.5]烷-7,9-二酮 $(2,1 \mathrm{mmol})$, 醛 $(3,1 \mathrm{mmol})$, 吲哚 $(\mathbf{1}, 1 \mathrm{mmol})$ 和 $\mathrm{ChCl} / \mathrm{Ox}(1 \mathrm{~mL})$, 磁力搅拌 $20 \sim 40$ $\min . \mathrm{TLC}$ 检测至反应完成后, 向反应试管中加入乙酸 乙酯 $(4 \mathrm{~mL})$ 和水 $(1 \mathrm{~mL})$, 有机相经水洗, 无水硫酸钠干 燥, 旋转蒸发除去有机溶剂后得粗产物. 粗产物经 $95 \%$ 乙醇重结晶得到纯品 $\mathbf{4 a \sim 4 1}$, 产率 70\% 97\%. 将水相 于 $60{ }^{\circ} \mathrm{C}$ 下减压 $(10 \mathrm{mmHg}$ )处理 $30 \mathrm{~min}$ 后即可回收低共 熔溶剂. 所有螺环吲哚化合物均为未知化合物, 其结构 经 IR, ${ }^{1} \mathrm{H} \mathrm{NMR},{ }^{13} \mathrm{C} \mathrm{NMR}$ 和 HRMS 确证. 产物理化数 据如下:

5-[(3-吲哚基)-苯甲基]-6,10-二氧杂螺[4.5]十烷-7,9二酮(4a): 白色固体, 产率 95\%. ${ }^{1} \mathrm{H}$ NMR (400 MHz, DMSO-d $\left.d_{6}\right) \delta: 10.99(\mathrm{~s}, 1 \mathrm{H}), 7.40 \sim 7.11(\mathrm{~m}, 8 \mathrm{H}), 7.08 \sim$ $7.00(\mathrm{~m}, 1 \mathrm{H}), 6.92 \sim 6.85(\mathrm{~m}, 1 \mathrm{H}), 5.37(\mathrm{~s}, 1 \mathrm{H}), 5.17(\mathrm{~s}$, $1 \mathrm{H}), 2.34 \sim 2.27(\mathrm{~m}, 2 \mathrm{H}), 2.02(\mathrm{~s}, 2 \mathrm{H}), 1.82 \sim 1.64(\mathrm{~m}$, $4 \mathrm{H}) ;{ }^{13} \mathrm{C}$ NMR (100 MHz, DMSO- $\left.d_{6}\right) \delta: 165.97,165.44$, $141.74,136.29,129.44,128.14,127.65,126.68,124.33$, $121.56,119.03,114.09,113.86,111.88,53.19,38.64$, 37.83, 23.98, 22.41; IR (KBr) $v_{\max }: 3472,2952,2880$, 1787, 1752, 1496, 1452, 1344, 1307, 1251, 1206, 1114, 998, 956, 749; HRMS calcd for $\mathrm{C}_{23} \mathrm{H}_{21} \mathrm{NO}_{4} \mathrm{Na}[\mathrm{M}+$ $\mathrm{Na}]^{+}$398.1363, found 398.1369.

5-[(3-吲哚基)-对硝基苯甲基]-6,10-二氧杂螺 [4.5] 十烷-7,9-二酮(4b): 黄色固体, 产率 97\%. ${ }^{1} \mathrm{H}$ NMR (400 $\left.\mathrm{MHz}, \mathrm{DMSO}-d_{6}\right) \delta: 11.13(\mathrm{~s}, 1 \mathrm{H}), 8.14(\mathrm{~d}, J=8.20 \mathrm{~Hz}$, 2H), 7.66 (d, $J=7.90 \mathrm{~Hz}, 2 \mathrm{H}), 7.43$ (s, 1H), 7.27 (d, $J=$ $8.30 \mathrm{~Hz}, 2 \mathrm{H}), 7.09$ (t, $J=7.20 \mathrm{~Hz}, 1 \mathrm{H}), 6.93$ (t, $J=7.20$ $\mathrm{Hz}, 1 \mathrm{H}), 5.49(\mathrm{~s}, 1 \mathrm{H}), 5.37(\mathrm{~s}, 1 \mathrm{H}), 2.39 \sim 2.37(\mathrm{~m}, 2 \mathrm{H})$, $2.11(\mathrm{~s}, 2 \mathrm{H}), 1.80$ (d, $J=6.40 \mathrm{~Hz}, 2 \mathrm{H}), 1.73(\mathrm{~d}, J=6.20$ $\mathrm{Hz}, 2 \mathrm{H}) ;{ }^{13} \mathrm{C}$ NMR (100 MHz, DMSO- $\left.d_{6}\right) \delta: 169.47$, 
$163.34,151.64,146.33,145.35,136.49,129.89,127.78$, $126.68,123.65,122.60,121.69,119.09,118.83,115.44$, $111.87,57.48,42.34,38.21,37.19,24.52,23.07$; IR (KBr) $v_{\max }: 3439,2967,2874,1791,1752,1514,1460,1348$, 1310, 1229, 1209, 1108, 964, 951, 734; HRMS calcd for $\mathrm{C}_{23} \mathrm{H}_{20} \mathrm{~N}_{2} \mathrm{O}_{6} \mathrm{Na}[\mathrm{M}+\mathrm{Na}]^{+}$443.1214, found 443.1220.

5-[(3-吲哚基)-对氟苯甲基]-6,10-二氧杂螺[4.5]十 烷-7,9-二酮(4c): 白色固体，产率 $88 \% ;{ }^{1} \mathrm{H}$ NMR (400 MHz, DMSO- $\left.d_{6}\right) \delta: 11.00(\mathrm{~s}, 1 \mathrm{H}), 7.36(\mathrm{~d}, J=8.20 \mathrm{~Hz}$, $3 \mathrm{H}), 7.22 \sim 7.12(\mathrm{~m}, 2 \mathrm{H}), 7.04(\mathrm{t}, J=8.90 \mathrm{~Hz}, 3 \mathrm{H}), 6.93 \sim$ $6.83(\mathrm{~m}, 1 \mathrm{H}), 5.34(\mathrm{~s}, 1 \mathrm{H}), 5.17(\mathrm{~d}, J=3.00 \mathrm{~Hz}, 1 \mathrm{H})$, $2.35 \sim 2.29(\mathrm{~m}, 2 \mathrm{H}), 2.05(\mathrm{~s}, 2 \mathrm{H}), 1.82 \sim 1.65(\mathrm{~m}, 4 \mathrm{H}) ;{ }^{13} \mathrm{C}$ NMR (100 MHz, DMSO- $\left.d_{6}\right) \delta$ : 165.44, 164.84, 159.56, $137.26,135.82,131.02,130.94,126.99,123.64,121.13$, $118.48,114.35,114.15,113.64,113.20,111.42,107,12$, $52.71,38.35,38.15,37.25,23.51,21.88$; IR (KBr) $v_{\max }$ : 3471, 2950, 2880, 1784, 1750, 1609, 1511, 1458, 1339, 1307, 1204, 1131, 998, 963, $749 \mathrm{~cm}^{-1}$; HRMS calcd for $\mathrm{C}_{23} \mathrm{H}_{21} \mathrm{FNO}_{4}[\mathrm{M}+\mathrm{H}]^{+}$394.1449, found 394.1456.

5-[(3-吲哚基)-对氯苯甲基]-6,10-二氧杂螺[4.5]十 烷-7,9-二酮(4d): 棕色固体, 产率 92\%; ${ }^{1} \mathrm{H}$ NMR (400 $\left.\mathrm{MHz}, \mathrm{CDCl}_{3}\right) \delta: 8.20(\mathrm{~s}, 1 \mathrm{H}), 7.44 \sim 7.38(\mathrm{~m}, 4 \mathrm{H}), 7.33$ (s, $1 \mathrm{H}), 7.24(\mathrm{~d}, J=6.9 \mathrm{~Hz}, 3 \mathrm{H}), 7.10(\mathrm{t}, J=7.1 \mathrm{~Hz}, 1 \mathrm{H}), 5.62$ $(\mathrm{s}, 1 \mathrm{H}), 4.30(\mathrm{~s}, 1 \mathrm{H}), 2.22 \sim 2.08(\mathrm{~m}, 4 \mathrm{H}), 1.95 \sim 1.81(\mathrm{~m}$, $4 \mathrm{H}) ;{ }^{13} \mathrm{C} \mathrm{NMR}\left(100 \mathrm{MHz}, \mathrm{CDCl}_{3}\right) \delta: 165.31,163.37$, $138.36,135.80,134.87,132.80,130.71,128.28,123.85$, $122.46,119.86,118.84,114.12,111.19,52.89,39.18$, $38.53,37.56,24.17,23.38$; IR (KBr) $v_{\max }: 3471,2981$, 2880, 1786, 1752, 1621, 1492, 1457, 1343, 1307, 1205, 1104, 1017, 999, 961, 746; HRMS calcd for $\mathrm{C}_{23} \mathrm{H}_{20} \mathrm{ClN}-$ $\mathrm{O}_{4} \mathrm{Na}[\mathrm{M}+\mathrm{Na}]^{+}$432.0973, found 432.0979.

5-[(3-吲哚基)-对溴苯甲基]-6,10-二氧杂螺 [4.5]十 烷-7,9-二酮(4e): 白色固体, 产率 91\%; ${ }^{1} \mathrm{H}$ NMR (400 MHz, DMSO-d $\left.d_{6}\right) \delta: 11.05(\mathrm{~s}, 1 \mathrm{H}), 7.45 \sim 7.39(\mathrm{~m}, 3 \mathrm{H})$, $7.33(\mathrm{~d}, J=7.60 \mathrm{~Hz}, 2 \mathrm{H}), 7.23(\mathrm{~s}, 2 \mathrm{H}), 7.08(\mathrm{t} J=6.80 \mathrm{~Hz}$, $1 \mathrm{H}), 6.94 \sim 6.90(\mathrm{~m}, 1 \mathrm{H}), 5.36(\mathrm{~s}, 1 \mathrm{H}), 5.23(\mathrm{~s}, 1 \mathrm{H}), 2.36 \sim$ $2.31(\mathrm{~m}, 2 \mathrm{H}), 2.08(\mathrm{~s}, 2 \mathrm{H}), 1.80 \sim 1.72(\mathrm{~m}, 4 \mathrm{H}) ;{ }^{13} \mathrm{C} \mathrm{NMR}$ (100 MHz, DMSO- $\left.d_{6}\right) \delta: 169.03,162.87,142.44,136.11$, $130.79,130.47,126.35,121.65,121.10,119.13,118.45$, $115.85,111.73,111.32,57.51,41.59,37.71,36.76,23.07$, 22.64,; IR (KBr) $v_{\max }: 3471,2982,2880,1785,1751,1621$, 1489, 1456, 1344, 1306, 1206, 1105, 1077, 1014, 998, 961, 746; HRMS calcd for $\mathrm{C}_{23} \mathrm{H}_{20} \mathrm{BrNO}_{4} \mathrm{Na}[\mathrm{M}+\mathrm{Na}]^{+}$ 476.0468, found 476.0476 .

5-[(3-吲哚基)-对甲基苯甲基]-6,10-二氧杂螺[4.5] 十烷-7,9-二酮(4f)：淡红色固体，产率 $85 \% ;{ }^{1} \mathrm{H}$ NMR (400 MHz, DMSO- $\left.d_{6}\right) \delta: 11.00(\mathrm{~s}, 1 \mathrm{H}), 7.38$ (s, 1H), 7.23 $(\mathrm{s}, 4 \mathrm{H}), 7.05(\mathrm{~s}, 3 \mathrm{H}), 6.90(\mathrm{~s}, 1 \mathrm{H}), 5.36(\mathrm{~s}, 1 \mathrm{H}), 5.15(\mathrm{~s}$, 1H), 2.27 (s, 2H), 2.24 (s, 3H), 2.05 (s, 2H), 1.75 (d, J= $27.7 \mathrm{~Hz}, 4 \mathrm{H}) ;{ }^{13} \mathrm{C}$ NMR (100 MHz, DMSO- $\left.d_{6}\right) \delta: 169.76$, $169.60,140.34,138.82,136.51,135.41,131.72,128.98$, $128.58,126.91,123.58,121.44,120.20,118.76,117.93$, $117.07,113.14,111.71,58.19,42.17,38.25,38.02,23.18$, 22.86, 20.99; IR (KBr) $v_{\max }: 3472,2951,2881,1786,1752$, 1653, 1559, 1515, 1457, 1340, 1306, 1204, 1105, 999, 961, 746; HRMS calcd for $\mathrm{C}_{24} \mathrm{H}_{23} \mathrm{NO}_{4} \mathrm{Na}[\mathrm{M}+\mathrm{Na}]^{+}$412.1519, found 412.1525 .

5-[(3-吲哚基)-对甲氧基苯甲基]-6,10-二氧杂螺 [4.5]烷-7,9-二酮(4g): 棕色固体，产率 74\%; ${ }^{1} \mathrm{H}$ NMR $\left(400 \mathrm{MHz}, \mathrm{DMSO}-d_{6}\right) \delta: 10.95$ (s, $\left.1 \mathrm{H}\right), 7.35$ (d, $J=7.90$ $\mathrm{Hz}, 1 \mathrm{H}), 7.28 \sim 7.18(\mathrm{~m}, 3 \mathrm{H}), 7.16(\mathrm{~d}, J=8.0 \mathrm{~Hz}, 1 \mathrm{H})$, $7.06 \sim 7.00(\mathrm{~m}, 1 \mathrm{H}), 6.87(\mathrm{t}, J=7.50 \mathrm{~Hz}, 1 \mathrm{H}), 6.78(\mathrm{~d}, J=$ $8.50 \mathrm{~Hz}, 2 \mathrm{H}), 5.31(\mathrm{~s}, 1 \mathrm{H}), 5.10(\mathrm{~d}, J=2.80 \mathrm{~Hz}, 1 \mathrm{H}), 3.69$ $(\mathrm{s}, 3 \mathrm{H}), 2.36 \sim 2.20(\mathrm{~m}, 2 \mathrm{H}), 2.02(\mathrm{~s}, 2 \mathrm{H}), 1.81 \sim 1.63(\mathrm{~m}$, $4 \mathrm{H}) ;{ }^{13} \mathrm{C}$ NMR (100 MHz, DMSO- $\left.d_{6}\right) \delta: 166.06,165.38$, $158.19,136.32,133.43,130.69,127.53,124.19,121.51$, $119.06,118.87,114.25,114.02,113.50,111.85,55.38$, $53.24,39.10,38.63,37.84,23.98,22.41$; IR (KBr) $v_{\max }$ : 3472, 2958, 2881, 1785, 1751, 1614, 1516, 1457, 1339, 1306, 1262, 1225, 1180, 1104, 1034, 998, 962, 747; HRMS calcd for $\mathrm{C}_{24} \mathrm{H}_{23} \mathrm{NO}_{5} \mathrm{~K}[\mathrm{M}+\mathrm{K}]^{+} 444.1208$, found 444.1212.

5-[(3-吲哚基)-邻氟苯甲基]-6,10-二氧杂螺[4.5]古 烷-7,9-二酮(4h): 淡红色固体，产率 91\%; ${ }^{1} \mathrm{H}$ NMR (400 MHz, DMSO-d $\left.d_{6}\right) \delta: 11.02(\mathrm{~s}, 1 \mathrm{H}), 7.42 \sim 7.25(\mathrm{~m}, 3 \mathrm{H})$, $7.25 \sim 7.20(\mathrm{~m}, 1 \mathrm{H}), 7.19 \sim 7.12(\mathrm{~m}, 2 \mathrm{H}), 7.05(\mathrm{dd}, J=$ 14.90, $7.30 \mathrm{~Hz}, 2 \mathrm{H}), 6.92(\mathrm{t}, J=7.40 \mathrm{~Hz}, 1 \mathrm{H}), 5.56(\mathrm{~d}, J=$ $3.80 \mathrm{~Hz}, 1 \mathrm{H}), 4.97(\mathrm{~d}, J=4.00 \mathrm{~Hz}, 1 \mathrm{H}), 2.40 \sim 2.22(\mathrm{~m}$, $2 \mathrm{H}), 2.09 \sim 2.04(\mathrm{~m}, 2 \mathrm{H}), 1.80 \sim 1.62(\mathrm{~m}, 4 \mathrm{H}) ;{ }^{13} \mathrm{C} \mathrm{NMR}$ $\left(100 \mathrm{MHz}, \mathrm{DMSO}-d_{6}\right) \delta: 165.54,165.42,159.33,136.26$, $132.04,128.84,128.74,127.26,124.18,121.72,119.12$, $118.74,115.43,115.20,114.26,113.02,111.97,51.58$, $38.73,38.22,37.86,33.37,23.15,22.39$; IR (KBr) $v_{\max }$ : 3430, 2985, 2892, 1790, 1752, 1490, 1456, 1344, 1291, 1287, 1200, 1117, 1055, 995, 960, 756, 747; HRMS calcd for $\mathrm{C}_{23} \mathrm{H}_{21} \mathrm{FNO}_{4}[\mathrm{M}+\mathrm{H}]^{+}$394.1449, found 394.1458.

5-[(3-吲哚基 $)$ - 邻甲氧基苯甲基 $]-6,10$-二氧杂螺 [4.5] 十烷-7,9-二酮(4i): 淡红色固体，产率 83\%; ${ }^{1} \mathrm{H}$ NMR (400 MHz, DMSO- $d_{6}$ ) $\delta: 10.98$ (s, 1H), 7.38 (d, $J=$ $7.70 \mathrm{~Hz}, 1 \mathrm{H}), 7.23$ (d, J=7.50 Hz, 1H), 7.17 $7.15(\mathrm{~m}$, 
2H), $7.09 \sim 6.94(\mathrm{~m}, 3 \mathrm{H}), 6.90(\mathrm{t}, J=7.10 \mathrm{~Hz}, 1 \mathrm{H}), 6.73$ $(\mathrm{d}, J=6.90 \mathrm{~Hz}, 1 \mathrm{H}), 5.56(\mathrm{~s}, 1 \mathrm{H}), 4.84(\mathrm{~s}, 1 \mathrm{H}), 3.86(\mathrm{~s}$, $3 \mathrm{H}), 2.28(\mathrm{~s}, 1 \mathrm{H}), 2.19 \sim 2.14(\mathrm{~m}, 1 \mathrm{H}), 2.08(\mathrm{~s}, 2 \mathrm{H}), 1.72$ $(\mathrm{s}, 4 \mathrm{H}) ;{ }^{13} \mathrm{C}$ NMR $\left(100 \mathrm{MHz}, \mathrm{DMSO}-d_{6}\right) \delta: 170.66$, 170.52, 161.97, 141.07, 136.07, 134.03, 132.78, 132.09, $128.83,126.43,125.44,123.66,121.41,118.70,116.68$, $116.53,115.56,60.76,55.90,43.47,43.00,39.52,27.92$, 27.21; IR (KBr) $v_{\max }: 3471,2981,2880,1786,1752,1621$, 1492, 1457, 1343, 1307, 1205, 1104, 1017, 999, 961, 746; HRMS calcd for $\mathrm{C}_{24} \mathrm{H}_{23} \mathrm{NO}_{5} \mathrm{~K}[\mathrm{M}+\mathrm{K}]^{+} 444.1208$, found 444.1217.

5-[(3-吲哚基)-2,5-二甲氧基苯甲基]-6,10-二氧杂螺 [4.5]十烷-7,9-二酮(4j): 黄色固体, 产率 $81 \% ;{ }^{1} \mathrm{H}$ NMR $\left(400 \mathrm{MHz}, \mathrm{CDCl}_{3}\right) \delta: 8.17(\mathrm{~s}, 1 \mathrm{H}), 7.52(\mathrm{~d}, J=8.00 \mathrm{~Hz}$, $1 \mathrm{H}), 7.34(\mathrm{~d}, J=8.10 \mathrm{~Hz}, 1 \mathrm{H}), 7.25(\mathrm{~s}, 1 \mathrm{H}), 7.16(\mathrm{t}, J=$ $7.30 \mathrm{~Hz}, 1 \mathrm{H}), 7.06(\mathrm{t}, J=7.20 \mathrm{~Hz}, 1 \mathrm{H}), 6.85 \sim 6.74(\mathrm{~m}$, 2H), $6.71(\mathrm{dd}, J=8.90,3.0 \mathrm{~Hz}, 1 \mathrm{H}), 5.70(\mathrm{~d}, J=3.10 \mathrm{~Hz}$, $1 \mathrm{H}), 4.50(\mathrm{~d}, J=3.20 \mathrm{~Hz}, 1 \mathrm{H}), 3.87(\mathrm{~s}, 3 \mathrm{H}), 3.58(\mathrm{~s}, 3 \mathrm{H})$, $2.25 \sim 2.04(\mathrm{~m}, 4 \mathrm{H}), 1.91 \sim 1.73(\mathrm{~m}, 4 \mathrm{H}) ;{ }^{13} \mathrm{C}$ NMR $(100$ $\mathrm{MHz}$, DMSO- $\left.d_{6}\right) \delta: 165.09,164.54,153.05,150.16$, $135.34,129.25,127.09,123.13,121.74,119.13,118.65$, $117.80,114.10,113.13,111.51,110.46,55.22,51.08$, $38.34,37.99,34.29,23.64,22.85,22.10$; IR (KBr) $v_{\max }$ : 3432, 3323, 2941, 2868, 1782, 1747, 1592, 1493, 1461, 1343, 1287, 1273, 1209, 1107, 1010, 968, 741; HRMS calcd for $\mathrm{C}_{25} \mathrm{H}_{25} \mathrm{NO}_{6} \mathrm{Na}[\mathrm{M}+\mathrm{Na}]^{+}$458.1574, found 458.1579 .

5-[(3-吲哚基)-间硝基苯甲基]-6,10-二氧杂螺 [4.5] 十烷-7,9-二酮(4k): 淡黄色固体, 产率 86\%; ${ }^{1} \mathrm{H}$ NMR (400 MHz, DMSO- $d_{6}$ ) $\delta: 11.11(\mathrm{~s}, 1 \mathrm{H}), 8.29(\mathrm{~s}, 1 \mathrm{H}), 8.05$ (d, $J=7.50 \mathrm{~Hz}, 1 \mathrm{H}), 7.85$ (d, $J=7.30 \mathrm{~Hz}, 1 \mathrm{H}), 7.54$ (t, $J=$ $7.90 \mathrm{~Hz}, 1 \mathrm{H}), 7.40(\mathrm{~d}, J=8.00 \mathrm{~Hz}, 1 \mathrm{H}), 7.25(\mathrm{~d}, J=7.20$ $\mathrm{Hz}, 2 \mathrm{H}), 7.08(\mathrm{t}, J=7.40 \mathrm{~Hz}, 1 \mathrm{H}), 6.91(\mathrm{t}, J=7.40 \mathrm{~Hz}$, $1 \mathrm{H}), 5.49(\mathrm{~s}, 1 \mathrm{H}), 5.36(\mathrm{~d}, J=2.50 \mathrm{~Hz}, 1 \mathrm{H}), 2.50 \sim 2.33$ (m, $2 \mathrm{H}), 2.10 \sim 2.09(\mathrm{~m}, 2 \mathrm{H}), 1.82 \sim 1.65(\mathrm{~m}, 4 \mathrm{H}) ;{ }^{13} \mathrm{C}$ NMR (100 MHz, DMSO- $d_{6}$ ) $\delta: 169.61,163.37,148.12$, 145.94, 136.53, 135.61, 129.95, 126.72, 123.21, 122.61, $121.69,119.10,118.84,115.74,112.20,111.88,57.70$, $42.13,38.20,37.19,23.14,22.81$; IR (KBr) $v_{\max }: 3432$, 3323, 2941, 2868, 1782, 1747, 1592, 1493, 1461, 1343, 1287, 1273, 1209, 1107, 1010, 968, 741; HRMS calcd for $\mathrm{C}_{23} \mathrm{H}_{20} \mathrm{~N}_{2} \mathrm{O}_{6} \mathrm{Na}[\mathrm{M}+\mathrm{Na}]^{+}$443.1214, found 443.1210.

5-[(3-吲哚基)-正丁基]-6,10-二氧杂螺[4.5]烷-7,9二酮(41): 白色固体, 产率 $72 \% ;{ }^{1} \mathrm{H}$ NMR $(400 \mathrm{MHz}$, $\left.\mathrm{CDCl}_{3}\right) \delta: 8.16(\mathrm{~s}, 1 \mathrm{H}), 7.67(\mathrm{~d}, J=7.80 \mathrm{~Hz}, 1 \mathrm{H}), 7.33$ (t,
$J=8.30 \mathrm{~Hz}, 1 \mathrm{H}), 7.30(\mathrm{~s}, 1 \mathrm{H}), 7.15(\mathrm{dt}, J=14.60,7.0 \mathrm{~Hz}$, $2 \mathrm{H}), 4.13(\mathrm{~s}, 1 \mathrm{H}), 3.79(\mathrm{~s}, 1 \mathrm{H}), 2.07 \sim 1.96(\mathrm{~m}, 2 \mathrm{H}), 1.93 \sim$ $1.43(\mathrm{~m}, 8 \mathrm{H}), 1.46 \sim 1.21(\mathrm{~m}, 2 \mathrm{H}), 0.91(\mathrm{t}, J=7.30 \mathrm{~Hz}$, $3 \mathrm{H}) ;{ }^{13} \mathrm{C}$ NMR $\left(100 \mathrm{MHz}, \mathrm{CDCl}_{3}\right) \delta: 165.98,165.54$, 135.72, 127.34, 123.93, 122.03, 119.65, 118.71, 115.11, $114.11,111.28,52.08,38.70,38.49,35.09,34.88,23.92$, 22.68, 21.43, 14.00; IR (KBr) $v_{\max }: 3478,2957,2875$, 1789, 1734, 1632, 1450, 1373, 1345, 1306, 1294, 1120, 1099, 1026, 991, 975, $740 \mathrm{~cm}^{-1}$; HRMS calcd for $\mathrm{C}_{20} \mathrm{H}_{23} \mathrm{NO}_{4} \mathrm{H}[\mathrm{M}+\mathrm{H}]^{+} 342.1700$, found 342.1706.

5-[(3-吲哚基)-正戊基]-6,10-二氧杂螺[4.5]十烷-7,9二酮(4m)：白色固体，产率 70\%; ${ }^{1} \mathrm{H}$ NMR $(400 \mathrm{MHz}$, $\left.\mathrm{CDCl}_{3}\right) \delta: 8.18(\mathrm{~s}, 1 \mathrm{H}), 7.67(\mathrm{~d}, J=7.80 \mathrm{~Hz}, 1 \mathrm{H}), 7.34(\mathrm{~d}$, $J=7.9 \mathrm{~Hz}, 1 \mathrm{H}), 7.29$ (d, $J=2.4 \mathrm{~Hz}, 1 \mathrm{H}), 7.21 \sim 7.11$ (m, 2H), $4.13 \sim 4.09(\mathrm{~m}, 1 \mathrm{H}), 3.80(\mathrm{~d}, J=2.50 \mathrm{~Hz}, 1 \mathrm{H}), 2.09 \sim$ $1.94(\mathrm{~m}, 2 \mathrm{H}), 1.94 \sim 1.60(\mathrm{~m}, 8 \mathrm{H}), 1.39 \sim 1.25(\mathrm{~m}, 4 \mathrm{H})$, $0.84(\mathrm{t}, J=6.80 \mathrm{~Hz}, 3 \mathrm{H}) ;{ }^{13} \mathrm{C}$ NMR $\left(100 \mathrm{MHz}, \mathrm{CDCl}_{3}\right) \delta$ : $165.42,164.97,135.20,126.82,123.37,121.54,119.16$, $118.21,114.71,113.58,110.72,51.56,38.19,38.02,34.66$, $32.08,29.96,23.41,22.17,22.09,13.49$; IR (KBr) $v_{\max }$ : 3386, 2956, 2851, 1786, 1748, 1572, 1458, 1433, 1343, 1294, 1195, 1130, 1096, 1010, $976739 \mathrm{~cm}^{-1}$; HRMS calcd for $\mathrm{C}_{21} \mathrm{H}_{26} \mathrm{NO}_{4}[\mathrm{M}+\mathrm{H}]^{+}$356.1856, found 356.1859 .

5-(2-硝基苯基亚甲基)-6,10-二氧杂螺[4.5]十烷-7,9二酮(4o): 黄色固体, 产率 95\%; ${ }^{1} \mathrm{H}$ NMR $(400 \mathrm{MHz}$, $\left.\mathrm{CDCl}_{3}\right) \delta: 8.75(\mathrm{~s}, 1 \mathrm{H}), 8.28(\mathrm{~d}, J=8.20 \mathrm{~Hz}, 1 \mathrm{H}), 7.74(\mathrm{t}$, $J=7.50 \mathrm{~Hz}, 1 \mathrm{H}), 7.65(\mathrm{t}, J=7.70 \mathrm{~Hz}, 1 \mathrm{H}), 7.50(\mathrm{~d}, J=$ $7.60 \mathrm{~Hz}, 1 \mathrm{H}), 2.35 \sim 2.11(\mathrm{~m}, 4 \mathrm{H}), 1.89(\mathrm{~s}, 4 \mathrm{H}),{ }^{13} \mathrm{C} \mathrm{NMR}$ $\left(100 \mathrm{MHz}, \mathrm{CDCl}_{3}\right) \delta: 162.09,159.61,155.51,146.36$, $133.87,130.97,130.37,129.77,124.83,118.54,114.52$, 38.60, 23.25; IR (KBr) $v_{\max }: 2963,2908,1765,1736,1625$, 1604, 1524, 1363, $800 \mathrm{~cm}^{-1}$; HRMS calcd for $\mathrm{C}_{15} \mathrm{H}_{13} \mathrm{~N}$ $\mathrm{O}_{6} \mathrm{Na}[\mathrm{M}+\mathrm{Na}]^{+}$326.0635, found 326.0642 .

辅助材料(Supporting Information) 化合物 $\mathbf{4 a} \sim 41$ 的 ${ }^{1} \mathrm{H}$ NMR, ${ }^{13} \mathrm{C}$ NMR 谱图. 这些材料可以免费从本刊网站 (http://sioc-journal.cn/)上下载.

\section{References}

[1] Tietze, L. F.; Brasche, G.; Gericke, K. M. Dmino Reactions in Organic Synthesis, Weinheim, Wiley-VCH, 2006.

[2] Feng, Y.-D.; Zhang, H.; Cheng, G.-L.; Cui, X.-L. Chin. J. Org. Chem. 2014, 34, 1499 (in Chinese) (冯亚栋, 张红, 程国林, 崔秀灵, 有机化学, 2014, 34, 1499.)

[3] Yang, D.-L.; Li, J.-R.; Sun, K.-N.; Lu, H.-Y.; Liu, M.-X.; Shi, D.-X. Chin. J. Org. Chem. 2013, 33, 2341 (in Chinese). (杨德利, 李加荣, 孙克宁, 路红燕, 刘明星, 史大昕, 有机化学, 2013, 33, 2341.) 
[4] Morteza, S. Chem. Rev. 2012, 112, 3508.

[5] Feng, X.; Wang, J.-J.; Lin, W.; Zhang, J.-J.; Huang, Z.-B.; Shi, D.-Q. Chin. J. Chem. 2014, 32, 889.

[6] Wu, L.; Chen, P.-H.; Liu, G.-S.; Huang, Z.-B. Chin. J. Chem. 2014, 32,681 .

[7] Epifano, F.; Genovese, S.; Rosati, O.; Tagliapietra, S.; Pelucchini, C.; Curini, M. Tetrahedron Lett. 2011, 52, 568.

[8] Ge'rard, S.; Renzetti, A.; Lefevre, B.; Fontana, A.; Maria, P.; Sapi, P. Tetrahedron 2010, 66, 3065.

[9] Armstrong, E.-L.; Grover, H.-K.; Kerr, M.-A. J. Org. Chem. 2013, $78,10534$.

[10] Cochard, F.; Laronze, M.; Sigaut, P.; Sapi, J.; Laronze, J.-Y. Tetrahedron Lett. 2004, 45, 1703.

[11] Oikawa, Y.; Hirasawa, H.; Yonemitsu, O. Tetrahedron Lett. 1978, 19, 1759.

[12] Nemes, C.; Jeannin, L.; Sapi, J.; Laronze, M.; Seghir, H.; Auge, F.; Laronze, J.-Y. Tetrahedron 2000, 56, 5479.

[13] Wang, H.-Y.; Li, L.-L.; Li, W.; Huang, Z.-B.; Shi, D.-Q. Chin. J. Org. Chem. 2013, 33, 1616 (in Chinese). (汪辉员, 李丽丽, 林伟, 黄志斌, 史达清, 有机化学, 2013, 33, 1616.)

[14] Renzetti, A.; Dardennes, E.; Fontana, A.; Maria, P.-D.; Sapi, J.; Gerard, S. J. Org. Chem. 2008, 73, 6824.

[15] Chandrasekhar, S.; Patro, V.; Reddy, G.-P.; Grée, R. Tetrahedron Lett. 2012, 53, 6223.
[16] Qu, Y.-Y.; Ke, F.; Zhou, L.; Li, Z.-K.; Xiang, H.-F.; Wu, D.; Zhou, X.-G. Chem. Commun. 2011, 47, 3912.

[17] Liu, B.-M.; Zhang, D.-L.; Guo, X.-M.; Li, H.-Z. Chin. J. Synth. Chem. 2004, 12, 505 (in Chinese). (刘卉闵, 张冬暖, 果秀敏, 李惠章, 合成化学, 2004, 12, 505.)

[18] Wang, C.; Zhang,Y.-Q.; Li, G.-S.; Li, J.-C.; Li, X.-L. Chin. J. Org. Chem. 2003, 23, 1416 (in Chinese).

(王春, 张英群, 李贵深, 李敬慈, 李晓陆, 有机化学, 2003, 23, 1416.)

[19] Zhang, Q.-H.; Vigier, K.-O.; Royer, S.; Jerome, F. Chem. Soc. Rev. 2012, 41, 7108.

[20] Ruß, C.; König, B. Green Chem. 2012, 14, 2969.

[21] Zhang, Y.-Y.; Lu, X.-H.; Hong, X.; Shi, Y.-J.; Jie, X.-Y. Prog. Chem. 2013, 25, 881 (in Chinese).

(张盈盈, 陆小华, 冯新, 史以俊, 吉晓燕, 化学进展, 2013, 25, 881.)

[22] Handy, S.; Westbrook, N.-M. Tetrahedron Lett. 2012, 55, 4969.

[23] Yadav, U.-N.; Shankarling, G.-S. J. Mol. Liquids 2014, 191, 137.

[24] Hawkins, I.; Handy, S.-T. Tetrahedron 2013, 69, 9200.

[25] Azizi, N.; Dezfooli, S.; Hashemi, M.-M. J. Mol. Liquids 2014, 194, 62.

[26] Yan, N.; Xiong, B.; Liao, W.-L.; Xu, Z.-H. Chin. J. Org. Chem. 2010, 30, 1391 (in Chinese).

(严楠, 熊斌, 廖维林, 许招会, 有机化学, 2010, 30, 1391.) 